\title{
Pizarro Pedraza, Andrea (ed.) (2018) \\ Linguistics Taboo Revisited: Novel Insights from Cognitive Perspectives
}

BERLÍN

DE GRUYTER MOUTON

ISBN 978-3-11-058031-0

331 PÁGS.

La obra que reseñamos nos ofrece una visión del fenómeno del tabú desde una perspectiva cognitiva, basada, sobre todo, en la Semántica, pero ligada a otras ciencias, como la Pragmática o el Análisis del discurso, debido a su carácter universal. En esta obra se abordan conceptos como "eufemismo", "disfemismo" y "ortofemismo", con los que se pretende ampliar el campo de estudio de este fenómeno. En este sentido, en el prefacio y en la introducción, Allan y Pizarro Pedraza, respectivamente, abarcan la etimología del tabú y nos remontan a su origen. Procedente de las lenguas polinesias, el tabú se usaba para referirse a los rituales prohibidos de estas culturas, así como por el respeto y el miedo provocado por los poderes metafisicos. Hoy en día, se usa para evitar el empleo de vocabulario obsceno, ya que es imposible eliminarlo de nuestra lengua, por lo que se recurren a usos eufemísticos, ortofemísticos y disfemísticos.

Los primeros estudios del tabú se centraron en temas problemáticos como la sexualidad, la religión, la muerte, etc. y fueron estudiados desde la Antropología y la Etnografia. Más tarde, la Lingüistica y, en concreto, la Semántica léxica, se interesó por este fenómeno que antes no había sido abordado, según Pizarro Pedraza (p. 2) por la dificultad extralingüistica motivada por la estigmatización social y por la dificultad lingüística basada en su complejidad inherente. No obstante, esta autora defiende el estudio del tabú lingüistico porque "studying linguistsic taboo means understanding the systems of beliefs, the regimes of language, truth and knowledge" (p. 2).

Este volumen se compone de un total de quince capitulos, sirviendo el primero de ellos ("Lexicon, discourse and cognition: terminological delimitations in the conceptualizations of linguistic taboo") como introducción. En él, Casas Gómez nos proporciona una revisión del estado de la cuestión y una revisión teórica y terminológica sobre el tabú lingüístico y otros conceptos como "eufemismo", "disfemismo", "ortofemismo", "interdicción", que requieren de una precisión terminológica, pues se usan de forma inadecuada en investigaciones de carácter pragmático-discursivo. De esta manera, el autor describe las principales identificaciones y confusiones existentes entre estos conceptos, destacando que eufemismo y disfemismo suelen identificarse con "sustituto eufemístico" o "sustituto disfemístico", y con procesos de sustitución de temas prohibidos en la realidad. Además, defiende la existencia de usos eufemísticos y disfemísticos (p. 15) creados para ser usados en contextos determinados, puesto que no se puede afirmar la existencia de eufemismos o palabras eufemísticas. Además, hace especial hincapié en la evolución conceptual y terminológica de las definiciones del concepto de eu- 
femismo, haciendo un recorrido por diferentes autores y épocas, y concluyendo, desde una perspectiva cognitiva, con que el eufemismo debe permitir al hablante, en una situación comunicativa, producir atenuaciones o expresiones adecuadas al contexto (p. 28).

Los capitulos restantes se organizan en cuatro bloques de contenido: Construal, Cultural Conceptualization, Cognitive Sociolinguistics e Interdisciplinary Approaches. El primero de ellos recopila los siguientes tres capitulos: "The axiological and communicative potential of homosexual-related metaphors", "Metonymy-based euphemisms in war-related speeches by George W. Bush and Barack Obama" y "Ambiguity and vagueness as cognitive tools for euphemistic and politically correct speech", en los que se explican fenómenos lingüísticos vinculados al tabú: la metáfora, la metonimia, la ambigüedad y la vaguedad. En el primer capítulo de este bloque, Crespo-Fernández estudia metáforas sobre la homosexualidad a través del Corpus of Contemporary American English (COCA), centrándose en las utilizadas por cualquier persona, independientemente de su orientación sexual y las clasifica en container, journeys, women y animals, analizando, a través de ellas, usos eufemísticos, disfemísticos y cuasi-eufemísticos. En el segundo, Moritz examina expresiones eufemísticas basadas en metonimias producidas en los discursos politicos de George W. Busch y Barak Obama, superando estas a los eufemismos basados en metáforas, que es el fenómeno más usado en estos discursos. En el último, Chamizo Domínguez defiende la idea de que el lenguaje políticamente correcto se basa en el uso de eufemismos y en concreto de fenómenos lingüísticos como la vaguedad y la ambigüedad, conceptos que distingue desde una perspectiva cognitiva. Según este autor (p. 80), la vaguedad hace referencia a que el significado dado a una palabra ya está incluido en el significado de otra, mientras que una palabra ambigua se caracteriza por ser polisémica.

La segunda parte de esta obra, Cultural Conceptualization, está compuesta por seis capítulos que estudian la relación entre el tabú y la conceptualización cultural. En el primero, "Old age revolution in Australian English: Rethinking a taboo concept", Benczes, Burridge, Allan y Sharifian analizan expresiones relacionadas con la vejez en el inglés australiano, partiendo de un corpus que contiene periódicos australianos desde 1987 hasta 2014. El estudio de la edad en Australia es importante, porque tiene la tercera proporción de personas mayores de 65 años en todo el mundo. A través de este capitulo se ha producido una redefinición o reconceptualización de las palabras referidas a la edad, ya que se han manifestado mediante la creación de nuevos conceptos; la extensión categorial, expresiones o palabras que reemplazan a otras por su carácter malsonante (seniors Australian frente a older Australians, p. 109) y la emergencia de metáforas y esquemas culturales. Zawrotna, en el segundo capítulo, "Taboo subjects as insult intensifiers in Egyptian Arabic", estudia el uso del tabú en el árabe egipcio como intensificador de insulto en las categorias "familia", "sexualidad" y "religión”. Estos dominios son interesantes porque son temas sagrados o prohibidos para los egipcios. Sin embargo, estas palabras tabúes han experimen- 
tado un proceso de gramaticalización insertando significados nuevos, perdiendo así sus significados originales, pero conservando su carga emocional, lo que da lugar a una intensificación gradual, donde el dominio "religión" es el más intenso. Para poder analizar estos dominios, la autora se ha servido del material recopilado durante 2013-2016 en el Cairo y de otros recursos online, como la red social Facebook. Con estos materiales ha realizado un estudio sociolingüístico a 60 participantes de entre 20-35 años con el objetivo de evaluar la vulgaridad y la ofensa de estas expresiones en estas categorias. En el tercero, "Emotion concepts in context: Figurative conceptualizations of hayâ 'self-restaint' in Persian", Bakhtinar analiza el concepto de hayâ en Persia a través de factores cognitivos, sociales, culturales y pragmáticos-discursivos. Se sirve de un corpus de periódicos con el que estudia 150 ejemplos de expresiones y colocaciones con el objetivo de proporcionar una interpretación cultural del concepto y su relación con otros. En "A Cognitive Linguistics approach to menstrutation as a taboo in Gĩkũyũ", Gatambuki Gathigia, Ochieng Orwenjo y Wangeci Ndung'u nos ofrecen una visión del concepto de la "menstruación" en África. En su análisis, nos proporcionan eufemismos y mecanismos cognitivos (el uso de metáforas) en Gĩkũyũ para hacer referencia a este proceso fisiológico. En concreto, se recopila un total de 29 metáforas sobre la menstruación, que quedan divididas en menstruation is a period, menstruation is a visitor, menstruation is an indisposition, menstruation is a colour, y menstruation is a valuable possession. Este capítulo demuestra que características fisiológicas de la mujer, como la menstruación, son temas tabúes para determinadas zonas o regiones. En el quinto capitulo, "The socio-cognitive aspects of taboo in two cultures: A case study on Polish and British English", Kuzio propone un análisis contrastivo del tabú entre dos culturas: la cultura polaca y la cultura del inglés británico, haciendo especial hincapié en el contexto. La autora, con este estudio, demuestra que el tabú es un fenómeno cultural y que presenta diferencias en base a la lengua y a la cultura, ya que se sustituye por expresiones o palabras propias de jergas, sinónimos o metáforas (p. 181). En esta línea, se distribuyeron 80 cuestionarios a polacos y británicos de diferente edad y nivel educativo y ambos grupos respondieron de forma distinta desde una perspectiva cultural y lingüística, pues influía el contexto y el uso de la palabra tabú. El último capítulo de esta sección, "The influence of concpetual differences on processing taboo metaphors in the foreign laguage", nos muestra diferentes factores, como el contexto o la cultura, que influyen en el procesamiento de metáforas tabú en la adquisición de una segunda lengua (L2). Los autores, De Cock y Suñer, nos ofrecen los resultados obtenidos en un estudio realizado con franceses nativos de Bélgica, que estudian español como segunda lengua. En él se analizan metáforas relacionadas con la sexualidad y nos confirman la importancia del contexto para la compresión de las expresiones tabú metafóricas, pues algunos de los participantes presentaban relación cultural con el español, lo que les facilitaba la compresión de este tipo de metáforas (p. 218).

El tercer bloque, Cognitive Sociolinguistics, agrupa capítulos relacionados con la variación del tabú: "Why do the Dutch swear with diseases?", "Calling 
things by their name: Exploring the social meanings in the preference for sexual (in)direct construals" $y$ "The perception of the expresion of taboos: a sociolinguistic study". Ruette, en el primero de ellos, aborda el tema del tabú en el uso de palabrotas o palabras mal sonantes en las categorias "religión", "sexualidad", "enfermedades" y "relaciones familiares" mediante mensajes escritos en la red social Twitter por usuarios polacos. El autor, con esta investigación, quiere demostrar que una palabra malsonante no siempre implica una situación vergonzosa en el acto comunicativo, pues en él influyen muchos factores como el uso de la palabra o el contexto en el que se produce. Teniendo en cuenta estos factores, puede producirse una conexión social entre los interlocutores o, por el contrario, una agresión lingüística (p. 227). En el segundo capítulo, Pizarro Pedraza se centra en la variación lingüística de 906 expresiones o conceptos vinculados a la sexualidad, en concreto, en las partes del cuerpo y en la fisiología humana. Se basa en el corpus MadSex, creado por la propia autora en 2013, el cual divide en expresiones directas basadas en conceptos sexuales (vulva) y en expresiones indirectas (little cupcake) (p. 245). Con esta investigación, la autora expone que los interlocutores optan por el uso de expresiones directas y que la sexualidad sigue siendo un tema tabú en muchas sociedades, lo que implica el uso del tabú lingüístico. Además, considera que este término abarca dos tipos de fenómenos: el tabú referencial, que es "la expresión de conceptos pertenecientes a las palabras prohibidas o campos semánticos problemáticos" (p. 247), como es el caso de la sexualidad, tema tratado en este trabajo; y el tabú no referencial, que se basaria en el estudio de las palabrotas e insultos. El último capítulo de esta parte se basa en un estudio sociolingüístico sobre expresiones tabú en el español madrileño. A través de este estudio, Cestero Mancera nos confirma el uso de la variación lingüística para hacer referencia a palabras tabú, que dependen de las funciones discursivas y de la influencia de factores sociales como la edad, el sexo, el nivel educativo o la clase social. Además, nos demuestra que los hombres, y, sobre todo, los jóvenes, usan expresiones más directas y obvian los ortofemismos, lo que confirma la influencia de las variantes sociolingüísticas en el uso de estas expresiones.

La obra culmina con dos capítulos recopilados en el bloque Interdisciplinary Approaches: "Scrupulosity, sexual ruminations and cleaning in Obsessive-Compulsive Disorder" y "Sewaring as emotions acts", capítulos que investigan el fenómeno del tabú en pacientes con trastornos lingüísticos. En concreto, Ghassemzadeh, en el primero de ellos, abarca la naturaleza del tabú y el concepto de escrupulosidad en pacientes con trastorno obsesivocompulsivo. La autora, además, destaca el Macbeth effect (p. 304), que es el mecanismo que hace referencia a los conceptos relacionados con la "limpieza del alma", ya que existe una amenaza a la pureza moral al realizar emisiones malsonantes vinculadas a la sexualidad o al concepto de escrupulosidad. Por último, Ritz Finkelsteins, analiza el uso involuntario de palabrotas o palabras malsonantes en pacientes con el sindrome de Tourette (coprolalia y copropaxia). Se trata de un sindrome que se produce de forma automática y que se ve incrementado en situaciones "prohibidas". El autor 
afirma que estas situaciones comunicativas deben analizarse desde la pragmática cognitiva, porque solo se produce una agresión lingüística por parte del hablante cuando el oyente asocia el enunciado con su intención comunicativa (p. 324).

En resumen, se trata de una obra que aborda perspectivas multidisciplinares del fenómeno del tabú desde un enfoque cognitivo, que puede resultar de utilidad para los lectores interesados en el estudio de este fenómeno complejo vinculado al ámbito de la Pragmática, la Sociolingüística o el Análisis del discurso.

SARA Gómez Díaz Personal Joven Investigador Universidad de Cádiz Facultad de Filosofia y Letras Instituto de Lingüistica Aplicada Avenida Duque de Nájera, 16. Edificio Simón Bolivar, $2 .^{a}$ planta 11071 Cádiz Teléfono: +34 678769361 sara.gomez@uca.es

Fecha de Recepción Fecha de Publicación
$16 / 03 / 2020$

$01 / 12 / 2020$
DOI : http://dx.doi.org/10.25267/Pragmalinguistica.2020.i28.17 